Centre de Recherche en Économie et Statistique

CREST

Série des Documents de Travail

$n^{\circ} 2011-31$

\title{
Intertemporal Price Discrimination in Infinite \\ Horizon
}

\author{
L. WILNER ${ }^{1}$
}

Les documents de travail ne reflètent pas la position du CREST et n'engagent que leurs auteurs.

Working papers do not reflect the position of CREST but only the views of the authors.

1 CREST (INSEE), 15 boulevard Gabriel Péri, 92245 Malakoff, Cedex, France. 


\title{
Intertemporal price discrimination in infinite horizon
}

\author{
Lionel Wilner*
}

November 24, 2011

\begin{abstract}
Intertemporal price discrimination may be used by a durable-good monopoly under incomplete information, facing heterogeneous and more impatient consumers. We solve the self-selection problem in infinite horizon, characterize the optimal price policy with an ordinary differential equation depending on agents' patience and provide a condition for existence and uniqueness of the solution. In the uniform case, we extend Stokey (1979) by showing that as soon as the firm is not much more patient than consumers, uniform pricing is preferred to price discrimination. Besides, linear contracts are optimal if valuations are either uniform or Pareto distributed.
\end{abstract}

Keywords Intertemporal price discrimination, durable-good monopoly, nonlinear pricing, non-transferability.

JEL C61, D21, D42.

${ }^{*}$ CREST (INSEE), 15 bd Gabriel Peri, 92245 Malakoff Cedex, France. Email: wilner@ensae.fr. I am indebted to Xavier D'Haultfoeuille and Philippe Février who suggested me to write down this version of the model. I am grateful to Michael Visser for his very valuable remarks. I thank Eric Avenel, Philippe Choné, Pierre Fleckinger, François Le Grand and participants at the 2011 ASSET meeting for their comments. All remaining errors are mine. 


\section{Introduction}

Consider a monopolist who produces a durable good in some initial period and sells it over time to a set of consumers. In the first period, the monopolist is tempted to charge the static maximizing profits monopoly price. In the second period, the monopoly faces a residual demand and wishes to sell additional units to the remaining consumers at a lower price. This process repeats itself and leads to lower the price until it is profitable, i.e. until the competitive price is reached. The Coase (1972) conjecture states that consumers anticipate this and will postpone buying until the price falls to this level, which, in equilibrium, must occur at the beginning. Stokey (1981) has proven this conjecture by showing that the unique perfect rational expectations equilibrium is such that the monopoly sells immediately at the competitive price; consumers buy as soon as they can whenever it is worth purchasing. Rational expectations and the inability of the monopoly to commit are the key ingredients that drive the result.

Many papers have tried either to rationalize the Coase conjecture (see Gul et al. (1986) and Güth \& Ritzberger (1998) among others) or to explain how its undesirable consequence could be avoided. Solutions may consist in reducing the durability of the good through renting or leasing (Bulow (1982)); capacity constraints may also be invoked (see McAfee \& Wiseman (2007)). Another way of avoiding the Coase conjecture consists in assuming that the firm is credible and can commit from the beginning to stick to some price scheme, which will be the case here. Finding the optimal strategy of the monopoly is then a standard nonlinear pricing problem under incomplete information about consumer valuations. Stokey (1979) showed that if the monopoly is able to commit then the static monopoly price is charged in each period, which guarantees positive profits. All sales occur at the beginning of the game. This result relies, however, on the assumption that the firm and consumers have the same discount factor. It no longer holds when agents have different intertemporal preferences. For instance, when the firm is more patient, it sounds that she should be able to price-discriminate among consumers.

This point was made by Landsberger \& Meilijson (1985) who described the optimal price policy and proved that when the monopolist is more patient, intertemporal price discrimination becomes profitable in a finite-horizon setting. They tackled this issue by providing a qualitative description of the optimal sales strategy. First, the difference between discount factors accounts for non-transferability and generates intertemporal discrimination as soon as the firm is more patient than consumers. Second, this strategy turns out to generate higher revenues than uniform pricing. Indeed, a time-decreasing price schedule makes high-valuation consumers buy relatively early provided that it is 
not profitable for them to wait for a lower price. Imposing such an incentive constraint is however less costly for a monopoly that is more patient than consumers.

We propose to write down the pricing model in infinite horizon and to compute explicitly the price policy, which helps in deriving new optimality results. We allow for different intertemporal preferences: the firm and consumers may have distinct discount factors, which encompasses several cases treated by the literature. We resort to variational methods to overcome the issue of non-transferability with respect to money between the firm and consumers: since agents have distinct intertemporal preferences, they do not value transfers identically, which complicates the resolution of the model. The framework considered here contains previous models as special cases; addressing the issue of intertemporal price discrimination versus uniform pricing, it tends to reconcile different point of views, including numerous papers devoted to the durable-good monopoly and at least Stokey (1979), Landsberger \& Meilijson (1985), Salant (1989).

The next section presents the model in case when the monopoly is strictly more patient than consumers, though not infinitely patient. Section 3 is examines the case where valuations are uniformly distributed while Section 4 addresses the general case. Section 5 treats the limits of the model: the case where the monopoly is infinitely patient, as patient as consumers, or less patient than consumers. Section 6 concludes.

\section{Model}

A monopolist (Principal) produces a good that does not depreciate over time. It is durable in the sense that consumers may purchase it at most once. Time is continuously measured on $[0 ;+\infty]$. The marginal cost to produce the good is constant and normalized to zero. The firm discounts time $t$ with a factor $\rho$ by $e^{-\rho t}$.

The asymmetric information issue arises from the fact that the monopolist does not know consumers' private valuation for the good $\theta$. The distribution of $\theta$ is assumed to be common knowledge. Its density function $f($.$) is continuously differentiable and strictly$ positive almost everywhere over its support $[0, \bar{\theta}]=\Theta \subset \mathbb{R}^{+}$. Since the lowest reservation value is 0 , at any positive price, including the best single price, i.e. the static monopoly price, not all consumers buy and thus the market is not covered. We assume that the static monopoly price is well-defined: $\exists p^{m} \in(0,+\infty)$ such that $p^{m}=\frac{1-F\left(p^{m}\right)}{f\left(p^{m}\right)}$.

The problem of the firm consists in designing a direct truthful mechanism that implements the trade of one unit of the good. For this purpose, the monopoly offers a menu of contracts made up of a price and a time of purchase $\{p(\theta), \tau(\theta)\}$. Consumer $\theta$ reporting 
$\tilde{\theta}$ gets the utility:

$$
U(\theta, \tilde{\theta})=e^{-r \tau(\tilde{\theta})}[\theta-p(\tilde{\theta})] .
$$

The surplus $\theta-p(\tilde{\theta})$ is discounted by $e^{-r \tau(\tilde{\theta})}$, where $r$ is the consumer's discount rate. We assume for now that the monopolist is more patient than consumers, i.e. $\rho<r$. In Section 5, we discuss the cases $\rho=+\infty, \rho=r$ and $\rho>r$.

Consumers with valuation below a threshold $\theta_{0}$ are excluded from the market, where $\theta_{0}$ corresponds to the marginal consumer who is indifferent between purchasing or not:

$$
p\left(\theta_{0}\right)=\theta_{0}
$$

The physical constraint that the time of purchase is necessarily nonnegative imposes:

$$
\tau(\theta) \geq 0 \quad \forall \theta
$$

In a finite-horizon setting, this constraint would write $\tau(\theta) \in[0, T] \forall \theta$.

The individual rationality of consumer $\theta$ imposes that the price is no higher than $\theta$; similarly, the firm offers nonnegative prices, hence we restrict our attention to price schedules for which:

$$
p(\theta) \in[0, \theta] \quad \forall \theta \in[\underline{\theta} ; \bar{\theta}] \quad\left(I R_{\theta}\right)
$$

The mechanism is incentive-compatible if and only if:

$$
\theta=\arg \max _{\tilde{\theta}} \quad U(\theta, \tilde{\theta}) \quad \forall \theta \in[\underline{\theta} ; \bar{\theta}] \quad\left(I C_{\theta}\right)
$$

The first-order condition writes: $\left.\frac{\partial U}{\partial \tilde{\theta}}\right|_{\tilde{\theta}=\theta}=0$, i.e.:

$$
\dot{p}(\theta)-r \dot{\tau}(\theta) p(\theta)=-r \dot{\tau}(\theta) \theta \quad \forall \theta \in[\underline{\theta} ; \bar{\theta}]
$$

Given $\tau(\theta)$, this incentive constraint looks like a differential equation in $p($.$) with the$ initial condition (1). We can solve it to obtain an explicit relationship between $p(\theta)$ and $\tau(\theta):$

$$
p(\theta)=\theta-\int_{\theta_{0}}^{\theta} e^{r[\tau(\theta)-\tau(u)]} d u
$$

The firm's program writes:

$$
\max _{\left(p(\theta), \tau(\theta), \theta_{0}\right)} \int_{\theta_{0}}^{\bar{\theta}} e^{-\rho \tau(\theta)} p(\theta) f(\theta) d \theta \quad \text { s.t. } \quad(2),(3),(5)
$$


which reduces to:

$$
\max _{\left(\tau(\theta), \theta_{0}\right)} \int_{\theta_{0}}^{\bar{\theta}} e^{-\rho \tau(\theta)}\left[\theta-\int_{\theta_{0}}^{\theta} e^{r[\tau(\theta)-\tau(u)]} d u\right] f(\theta) d \theta \quad \text { s.t. }
$$

Note that the firm fixes the level of the marginal consumer type $\theta_{0}$. It may thus decide not to sell to all consumers by choosing $\theta_{0}>0$. Using the above maximization program in a finite horizon, ${ }^{1}$ Landsberger \& Meilijson (1985) show that intertemporal price discrimination achieves higher profits than uniform pricing when the firm is strictly more patient than consumers and when not all consumers buy at the best single price.

The program of the firm consists in optimizing with respect to a function; hence, we use variational calculus to solve this problem. Contrary to many problems in mechanism design, like Mirrlees (1971) for instance, agents have different intertemporal preferences; this property can be called non-transferability. Indeed, each trade of one unit at time $t$ is valued $e^{-\rho t}$ by the firm but $e^{-r t}$ by consumers. It requires especially to relax the usual assumption of quasi-linear preferences with respect to transfers, where transfers have respective coefficients -1 and +1 for the Principal and the Agent.

Letting $\lambda=\frac{\rho}{r} \in(0,1), y(\theta)=\int_{\theta_{0}}^{\theta} e^{-r \tau(u)} d u$ and $\dot{y}(\theta)=e^{-r \tau(\theta)}$, the previous program can be rewritten:

$$
\max _{\left(y(\theta), \theta_{0}\right)} \int_{\theta_{0}}^{\bar{\theta}} \dot{y}(\theta)^{\lambda}\left[\theta-\frac{y}{\dot{y}}(\theta)\right] f(\theta) d \theta \quad \text { s.t. } \quad\left\{\begin{array}{l}
\dot{y}(\theta) \in(0,1) \\
\dot{y}(\theta) \in[0, \theta]
\end{array}\right.
$$

To solve this constrained program, we will first solve the relaxed program and then check whether constraints are verified or not. The relaxed program can be written as:

$$
\max _{\theta_{0}} \max _{y(\theta)} \int_{\theta_{0}}^{\bar{\theta}} \mathcal{L}(\theta, y(\theta), \dot{y}(\theta)) d \theta
$$

where

$$
\mathcal{L}(\theta, y(\theta), \dot{y}(\theta))=\dot{y}(\theta)^{\lambda}\left[\theta-\frac{y(\theta)}{\dot{y}(\theta)}\right] f(\theta) .
$$

To make the resolution easier, the crucial point is to define $z(\theta)=\frac{y}{\dot{y}}(\theta) \in[0, \theta]$ which is simply consumer $\theta$ 's value of his surplus $\theta-p(\theta){ }^{2}$

Proposition 1 The solution $z($.$) verifies:$

$$
0=\dot{f}(\theta)[\lambda \theta+(1-\lambda) z(\theta)]+f(\theta)\left[2-(1-\lambda)[1-\dot{z}(\theta)]\left[2-\lambda+\lambda \frac{\theta}{z(\theta)}\right]\right]
$$

\footnotetext{
${ }^{1}$ that is, considering $\tau(\theta) \in[0, T] \forall \theta$ instead of constraint (2)

${ }^{2}$ From Equation (5) one has $p(\theta)=\theta-\frac{y(\theta)}{\dot{y}(\theta)}$.
} 
with the initial condition $z\left(\theta_{0}\right)=0$ stating that consumer $\theta_{0}$ 's surplus is zero.

Proof The stationary solutions of the Lagrangian (8) are given by the Euler-Lagrange equation, which boils down to the simple first-order differential Equation (9). The existence and the uniqueness of the function $z($.$) follow from the Cauchy-Lipschitz theorem.$ This characterizes completely $y($.$) up to y(1)$, or equivalently $\tau(1)$ that will be set to 0 by the impatient monopoly. Details are given in Appendix.

We study first the case when the distribution of valuations is uniform (Section 3), and then we analyze the general case (Section 4). Although we obtain explicitly the solution in the first case only, the variational approach used here allows us to characterize further the general case.

\section{The uniform distribution}

Consider the case where $\theta$ is uniformly distributed on $[0,1]$. Then we can state:

Proposition 2 The linear contract $p(\theta)=\left(1-K_{\lambda}\right) \theta$, with the corresponding schedule of purchases $\tau(\theta)=\frac{1-\frac{1}{K_{\lambda}}}{r} \log \theta$, and $\theta_{0}=0=\underline{\theta}$, maximizes the problem of the Principal with $K_{\lambda}=\left(\sqrt{\frac{\lambda}{2-\lambda}}-\lambda\right) \frac{1}{1-\lambda}$. Three situations may occur: indeed, $\exists 0<\underline{\lambda}<\bar{\lambda}<1$, such that:

- $\lambda \in(0, \underline{\lambda}]$ : the firm chooses to price discriminate, which is optimal

- $\lambda \in(\underline{\lambda}, \bar{\lambda})$ : the firm chooses to price discriminate, which is sub-optimal

- $\lambda \in[\bar{\lambda}, 1)$ : the firm chooses uniform pricing, which is optimal

Moreover, intertemporal price discrimination can be implemented by committing to charge the following price schedule:

$$
p(t)=\left(1-K_{\lambda}\right) e^{-\frac{K_{\lambda} r t}{1-K_{\lambda}}}
$$

Under uniform pricing, the monopoly charges $p^{m}=\frac{1}{2}$ and earns $\Pi^{m}=\frac{1}{4}$. Half of consumers buy immediately since it is not worth waiting: $\tau(\theta)=0$, and half of the market is excluded, consumers with a valuation less than $\frac{1}{2}$. Profits achieved with discrimination turn out to be $\Pi^{d}(\lambda)=\frac{1-K_{\lambda}}{2+\lambda\left(\frac{1}{K_{\lambda}}-1\right)}$. Furthermore, $\theta_{0}=0$ and $\tau(\theta)>0$ as soon as $\theta>\theta_{0}$, which means that everybody buys, though not immediately. This trade-off between impatience and rent extraction is depicted by Figure (1a) that compares $\Pi^{d}($.$) with \Pi^{m}$ : 
it is easy to check that $\Pi^{d}($.$) decreases with \lambda$ since the higher $\lambda$, the less patient the firm is with respect to consumers and thus she has less market power over them. There exists a threshold $\bar{\lambda} \approx 0.27$ such that if the firm is patient enough $(\lambda<\bar{\lambda})$, she will price discriminate and earn $\Pi^{d}(\lambda)$, while she will charge a uniform price and earn $\Pi^{m}$ otherwise. The latter case extends Stokey (1979) where the firm and consumers are equally patient $(\lambda=1)$ and in which the price stays at $p^{m}$ forever, purchases occurring immediately. This result holds in an infinite horizon even if the firm is strictly more patient than consumers, as soon as she is not too much patient. On the one hand, she can sell immediately to half of the market. On the other hand, she can extract more surplus to more consumers later on, and in an infinite horizon, the time of purchase can be very late, which is not in favor of price discrimination, contrary to what happens in finite horizon where price discrimination is profitable as soon as $\lambda<1$.

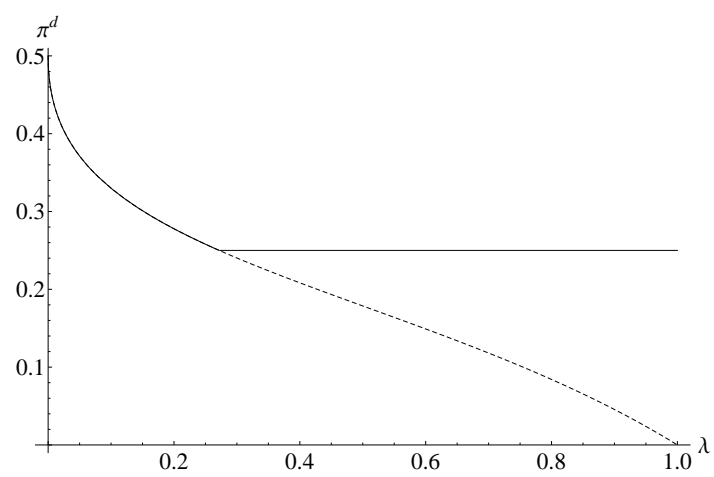

(a) profits

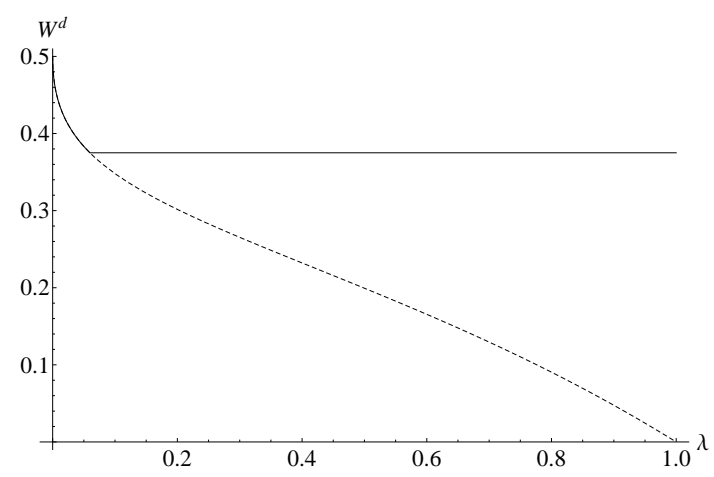

(b) welfare

Figure 1: Intertemporal price discrimination versus uniform pricing

Furthermore, the consumer surplus is always lower under discrimination, ${ }^{3}$ though the market is covered, contrary to what happens with uniform pricing. Indeed, the infinite horizon enables the firm to exploit as much as possible the fact that she may be just a little more patient than consumers, and to extract their rent. This phenomenon is large enough to compensate the gain incurred by the inclusion of half of the market.

Finally, comparing welfare under uniform pricing $\left(W^{m}=\frac{3}{8}\right)$ and price discrimination in Figure (1b) leads to determine a threshold of relative impatiences $\underline{\lambda} \approx 0.06$ above which uniform pricing does better than discrimination in terms of welfare. As a result, in the absence of costs, there is a range of impatiences $(\underline{\lambda}, \bar{\lambda})$ for which the monopoly chooses to price discriminate over time while uniform pricing is welfare-maximizing.

\footnotetext{
${ }^{3} \forall \lambda \in(0,1) \quad S^{d}(\lambda)<S^{m}(\lambda)=\frac{1}{8}$
} 
This specific example is helpful for obtaining results even in the case when distributions are not uniformly distributed. This is what we are going to study now.

\section{General distributions}

When the distribution of valuations is unknown, the solution is still characterized by (9) even though there is not always a closed-form solution.

First, we discuss the existence and the uniqueness of the solution to Equation (9).

Proposition 3 Sufficient conditions for the existence and the uniqueness of an extremum of the Lagrangian are given by $\dot{f}(0)=0$ and $\exists M>0$ such that $\left\|\frac{\dot{f}}{f}\right\|_{\infty} \leq M$.

Proof These conditions guarantee that the Cauchy-Lipschitz theorem applies and implies that Equation (9) admits a unique solution. By the strong Legendre condition, this extremum is a maximum. More details are given in Appendix.

Note that since the support of $f($.$) must be contained in a compact of [0 ;+\infty)$, or by extension $[0 ;+\infty)$, possible distribution functions are for instance the uniform, chi-square, Weibull, power, Gamma, Beta, Pareto or log-normal distributions. The condition $\dot{f}(0)=$ 0 rules out exponential distributions for which $f(\theta)=\lambda \exp (-\lambda \theta), \dot{f}(\theta)=-\lambda^{2} \exp (-\lambda \theta)$ and thus $\dot{f}(0)=-\lambda^{2}<0$. Similarly, it can easily be checked that a Weibull law meets this criterion if and only if it has a scale parameter strictly larger than 2; the same holds for Gamma and Beta distributions with shape parameters strictly larger than 2, and for a chi-square distribution with at least 2 degrees of freedom. Power distributions must also have a parameter strictly larger than 2 .

Second, we find that linear contracts correspond either to uniform or Pareto distributions of valuations, when allowing by extension for the support $\Theta$ to be $[\underline{\theta} ;+\infty)$ with $\underline{\theta}>0$.

Proposition 4 Linear contracts are optimal either if valuations are uniformly distributed over $[\underline{\theta} ; \bar{\theta}]$ or Pareto-distributed with shape parameter smaller than 1 over $[\underline{\theta} ;+\infty)$. The market is covered: $\theta_{0}=0 \leq \underline{\theta}$.

Several papers in the empirical taxation literature have found that income follows a distribution close to a Pareto distribution, which means that such an heterogeneity of valuations is a quite reasonable hypothesis in practice.

Third, it is possible to solve Equation (9) numerically for any given distribution satisfying $\left\|\frac{\dot{f}}{f}\right\|_{\infty}$ bounded and $\dot{f}(0)=0$, and to compute the solution, which cannot be linear 
unless $f($.$) is uniform or Pareto. The literature has already documented that the loss$ of using linear contracts as an approximation should not be large: as a matter of fact, D'Haultfoeuille \& Février (2011) find that this loss does not exceed $16 \%$ in a structural estimation. Indeed, Figure 2 shows that the optimal contract is not far from being linear.

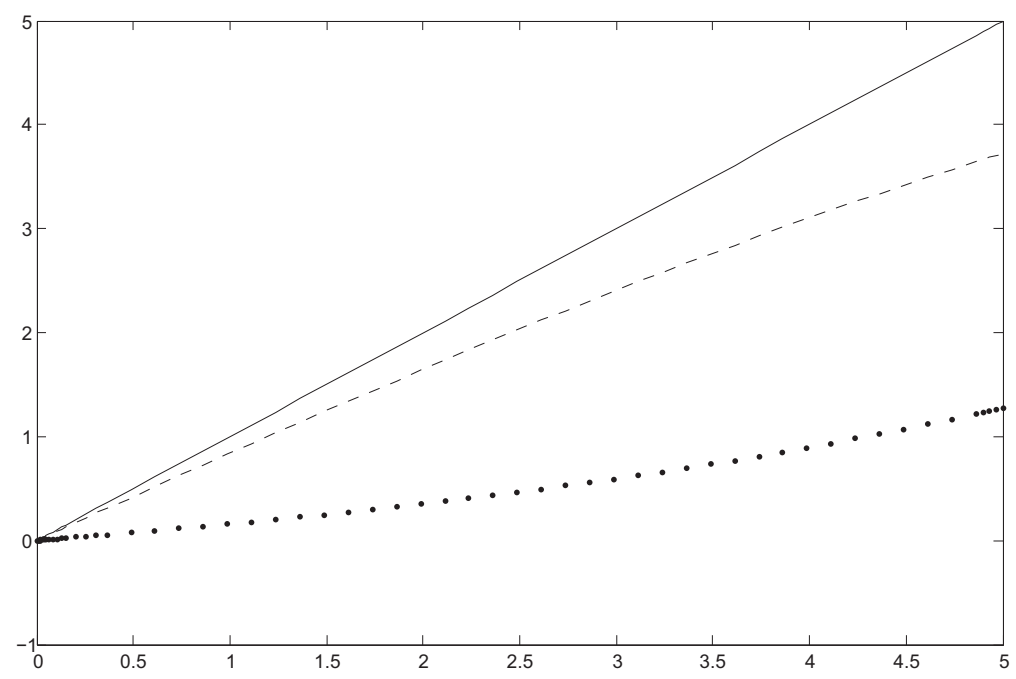

Figure 2: Optimal contract for a $\chi^{2}(2)$ distribution

Valuation (plain), price (dashed) and rent (dots) as functions of $\theta$

\section{Extensions}

Finally, we provide some insight on two limit cases of the model: $\lambda=0$ or $\lambda=1$. We discuss informally the case when $\lambda>1$.

\subsection{An infinitely patient monopoly: $\lambda=0$}

Proposition 1 can still be used to solve the case when the firm is infinitely patient, i.e. when $\lambda=\frac{\rho}{r}=0$. Regardless of how valuations are distributed, perfect price discrimination achieves full extraction of consumer surplus $\mathbb{E} \theta$ at infinity. The contract $(p(\theta)=\theta, \tau(\theta)=+\infty)$ is the unique solution to Equation (9), that rewrites $\dot{f}(\theta) z(\theta)+$ $2 f(\theta) \dot{z}(\theta)=0$, since the unique function verifying (9) with the initial condition $z\left(\theta_{0}\right)=0$ is $z(\theta)=0$. 


\subsection{Equally patient agents: $\lambda=1$}

The method used here can also be useful to understand the case when the firm and consumers are equally patient: $\lambda=\frac{\rho}{r}=1$. Stokey (1979) has proved that the price is constant and equal to $p^{m}$, such that sales occur immediately. Formally, the optimal contract involves $\left(p(\theta)=p^{m}, \tau(\theta)=0\right) \quad \forall \theta \geq p^{m}$ while $\tau(\theta)=+\infty \quad \forall \theta<p^{m}$, these consumers being excluded from the market. This contract corresponds to a corner solution where the constraint $\dot{y}(\theta) \in(0,1)$ is binding. The Euler-Lagrange equation writes here: $-f(\theta)=\frac{d}{d \theta}[\theta f(\theta)]$ and, by integration, depending on the sign of $1-F(\theta)-\theta f(\theta)$, might imply either that $\dot{y}=0$ or $\dot{y}=1$. To be precise, $\dot{y}=\mathbb{1}[1-F(\theta) \leq \theta f(\theta)]$, which leads to uniform pricing at $p^{m}$ for high valuation consumers whose $\theta \geq p^{m}$ and which excludes low valuation consumers with $\theta<p^{m}$. On the one hand, high valuation consumers buy immediately: $\tau(\theta)=0$, which corresponds to $\dot{y}(\theta)=1$ and $y(\theta)=\theta-p^{m}$. On the other hand, consumers whose valuation is smaller than $p^{m}$ never buy, $\dot{y}(\theta)=y(\theta)=0$.

\subsection{A more impatient monopoly: $\lambda>1$}

When the firm is less patient than consumers, she cannot do better than in the previous case: she is losing market power as time goes by, and prefers purchases to happen immediately. Thus, the outcome will be uniform pricing as previously, and formally, the same corner solution as before.

\section{Concluding remarks}

This note studies a common problem in the mechanism design literature, in which there is non-transferability in a dynamic setting stemming from the fact that agents may have different intertemporal preferences and thus discount the future differently. We provide a framework for price discrimination in infinite horizon that contains all cases evoked in the literature, when the firm is more/less (resp. as) patient than (resp. as) consumers, and that reconciles different points of views. First, we characterize the solution of the monopoly's problem through a simple differential equation; we provide a condition under which this equation admits a unique solution in the general case, i.e. for any distribution of valuations. Second, we find that optimal contracts are linear if and only if valuations are uniformly or Pareto-distributed. Third, when valuations are uniformly distributed, we show that, for intertemporal price discrimination to be profitable with respect to uniform pricing, the firm must be much more patient than consumers, not just more patient, which extends Stokey (1979); we quantify the corresponding threshold of patience that results from the trade-off between impatience and rent extraction. 


\section{Appendix: proofs}

\section{Proof of Proposition 1}

A necessary condition for optimization w.r.t. a function is given by the Euler-Lagrange equation:

$$
\frac{\partial \mathcal{L}}{\partial y}=\frac{d}{d \theta} \frac{\partial \mathcal{L}}{\partial \dot{y}}
$$

This equation defines the set of functions that are stationary points of the Lagrangian. Here, this equation boils down to a second-order nonlinear differential equation in $y($.$) :$

$$
0=\dot{f}(\theta)\left[\lambda \theta+(1-\lambda) \frac{y(\theta)}{y(\theta)}\right]+f(\theta)\left[2-(1-\lambda)\left[(2-\lambda) \frac{y(\theta)}{\dot{y}(\theta)} \frac{\ddot{y}(\theta)}{\dot{y}(\theta)}+\lambda \theta \frac{\ddot{y}(\theta)}{\dot{y}(\theta)}\right]\right]
$$

A sufficient condition for $y($.$) to be a maximum of Program (6) is given by the strong$ Legendre-Clebsch condition. This condition is the analogue of the second-order condition in a maximization problem w.r.t. a variable. It requires that the second derivative of the Lagrangian w.r.t. $\dot{y}($.$) be strictly negative: \frac{\partial^{2} \mathcal{L}}{\partial \dot{y}^{2}}<0$. Here, this condition is always satisfied since:

$$
\frac{\partial^{2} \mathcal{L}}{\partial \dot{y}^{2}}=-(1-\lambda) f(\theta) \dot{y}(\theta)^{\lambda-3}[\lambda \theta \dot{y}(\theta)+(2-\lambda) y(\theta)]<0
$$

because $\lambda \in(0,1), f($.$) is strictly positive almost everywhere on its support, \dot{y}(\theta) \in(0,1)$ and $y(\theta) \in[0, \theta]$.

\section{Proof of Proposition 2}

In case when $f(\theta)=1$, Equation (9) rewrites:

$$
2=(1-\lambda)[1-\dot{z}(\theta)]\left(2-\lambda+\lambda \frac{\theta}{z(\theta)}\right),
$$

which is a differential equation of the form $z=\theta f(\dot{z})$, i.e., a homogeneous Lagrange equation. A singular solution to equation (9) is given by $z(\theta)=K \theta$ - with $K \in[0,1]$ since by $(4), z(\theta) \in[0, \theta]$. However, there could exist other regular solutions to this equation but this is ruled out by the Cauchy-Lipschitz theorem which guarantees the existence and the uniqueness of the solution. To that purpose, let us show that conditions for Cauchy-Lipschitz are met.

First, we consider Equation (9) with the initial condition $z\left(\theta_{0}\right)=0$ coming from $y\left(\theta_{0}\right)=0$ and $\dot{y}\left(\theta_{0}\right)>0$, which defines a proper ordinary differential equation that can be rewritten as a Lagrange equation $\dot{z}(\theta)=g\left(\frac{z(\theta)}{\theta}\right)=h(z, \theta)$. 
Second, the solution $z($.$) must evolve in [0,1]$ because of individual rationality (3):

$$
z(\theta)=\theta-p(\theta) \in[0, \theta] \subset[0,1]
$$

and then $h(.,$.$) is continuous over its compact definition set:$

$$
\begin{aligned}
h:[0,1] \times\left[\theta_{0}, 1\right] & \longrightarrow \mathbb{R} \\
(z, \theta) & \longmapsto 1-\frac{\frac{2}{1-\lambda}}{\lambda \frac{\theta}{z}+2-\lambda}
\end{aligned}
$$

Third, a sufficient condition for $h(.,$.$) being Lipschitz is to have its first derivative h_{z}(.,$. being continuous and therefore bounded. One has:

$$
h_{z}(z, \theta)=-\frac{2}{1-\lambda} \frac{\lambda \frac{\theta}{z^{2}}}{\left[\lambda \frac{\theta}{z}+2-\lambda\right]^{2}}=-\frac{2 \lambda \theta}{1-\lambda} \frac{1}{[\lambda \theta+(2-\lambda) z]^{2}} .
$$

This expression can be bounded when $\theta>0$ :

$$
\left|h_{z}(z, \theta)\right| \leq \frac{2}{(1-\lambda) \lambda \theta} .
$$

Finally, if $\theta=0, h_{z}(z, 0)=0$.

As a result, the Cauchy-Lipschitz theorem states that there is a unique solution to Equation (9) that makes the profit extremal: $z(\theta)=K \theta$.

This extremum is a maximum by the strong Legendre-Clebsch condition already mentioned.

To fully characterize the solution, we find $K_{\lambda}$ by plugging $z(\theta)=K_{\lambda} \theta$ into Equation (9). $K_{\lambda}$ is the unique positive root of $(1-\lambda)(2-\lambda) X^{2}+2 \lambda(2-\lambda) X-\lambda(1-\lambda)=0$, which gives $K_{\lambda}=\left(\sqrt{\frac{\lambda}{2-\lambda}}-\lambda\right) \frac{1}{1-\lambda}$.

Next, from $z(\theta)=K_{\lambda} \theta=\frac{y(\theta)}{\dot{y}(\theta)}$, and since $\dot{y}(\theta)=e^{-r \tau(\theta)}$, we have:

- $y(\theta)=y(1) \theta^{\frac{1}{K_{\lambda}}}$

- $p(\theta)=\left(1-K_{\lambda}\right) \theta$

- $\dot{y}(\theta)=\frac{y(1)}{K_{\lambda}} \theta^{\frac{1}{K_{\lambda}}-1}$

- $\tau(\theta)=\frac{\log K_{\lambda}-\log y(1)}{r}+\frac{1-\frac{1}{K_{\lambda}}}{r} \log \theta$

All functions of interest are known up to $\tau(1)=-\frac{\log \dot{y}(1)}{r}=-\frac{\log \frac{y(1)}{K_{\lambda}}}{r}$. 
To see why $\tau(1)=0$, note that the firm's objective can be rewritten as:

$$
\max _{\tau(1), \theta_{0}} \int_{\theta_{0}}^{1}\left[e^{-r \tau(1)} \theta^{\frac{1}{K_{\lambda}}-1}\right]^{\lambda}\left(1-K_{\lambda}\right) \theta d \theta
$$

The solution is $\tau(1)=0$ and $\theta_{0}=0$ since $\lambda \in(0,1)$.

To conclude,

- $y(\theta)=K_{\lambda} \theta^{\frac{1}{K_{\lambda}}}$ is equal to the ex-post utility of $\theta$ reporting his true type

- $\dot{y}(\theta)=\theta^{\frac{1}{K_{\lambda}}-1} \in(0,1)$ almost everywhere; $\frac{y(\theta)}{\dot{y}(\theta)}=K_{\lambda} \theta \in[0, \theta]$

- $p(\theta)=\left(1-K_{\lambda}\right) \theta$

- $\tau(\theta)=\frac{1-\frac{1}{K_{\lambda}}}{r} \log \theta$

Profits are given by: $\Pi^{d}(\lambda)=\frac{1-K_{\lambda}}{2+\lambda\left(\frac{1}{K_{\lambda}}-1\right)}$.

$\tau($.$) being a one-to-one mapping, the corresponding pattern of prices over time is:$

$$
p(t)=\left(1-K_{\lambda}\right) e^{-\frac{r t}{\frac{1}{K_{\lambda}}-1}} .
$$

\section{Proof of Proposition 3}

Considering again Equation (9), the proof for existence and uniqueness is similar to the previous one but the function $h(.,$.$) writes more generally:$

$$
\begin{aligned}
h:[0,1] \times\left[\theta_{0}, \bar{\theta}\right] & \longrightarrow \mathbb{R} \\
(z, \theta) & \longmapsto 1-\frac{1}{1-\lambda} \frac{2+\frac{\dot{f}(\theta)}{f(\theta)}[\lambda \theta+(1-\lambda) z]}{\lambda \frac{\theta}{z}+2-\lambda}
\end{aligned}
$$

We have:

$$
h_{z}(z, \theta)=-\frac{1}{1-\lambda} \frac{1}{[(2-\lambda) z+\lambda \theta]^{2}}\left[2 \lambda \theta+\frac{\dot{f}(\theta)}{f(\theta)}\left\{(1-\lambda) z+[\lambda \theta+(1-\lambda) z]^{2}\right\}\right] .
$$

The absolute of this term is smaller than $\frac{2}{(1-\lambda) \lambda^{2} \theta^{2}}\left(1+\left\|\frac{\dot{f}}{f}\right\|_{\infty}\right)$ that is bounded as soon as $\theta \neq 0$ if $\left\|\frac{\dot{f}}{f}\right\|_{\infty}$ is bounded. If $\theta=0$,

$$
h_{z}(z, 0)=-\frac{\dot{f}(0)}{f(0)} \frac{1}{(2-\lambda)^{2}}\left(1-\lambda+\frac{1}{z}\right)
$$

that remains unbounded as $z$ approaches 0 unless $\dot{f}(0)=0$. 


\section{Proof of Proposition 4}

The sufficiency can be checked by replacing $f(\theta)$ in Equation (9) with $\frac{1}{\bar{\theta}-\underline{\theta}}$ (uniform) or with $\epsilon \underline{\theta}^{\epsilon} \theta^{-1-\epsilon}, \epsilon \in(0,1)$ (Pareto). The former case yields $K_{\lambda}$ of Proposition 2 while the latter case yields:

$$
K_{\lambda \epsilon}=\frac{\sqrt{\lambda(\epsilon-1)[\lambda(\epsilon+1)-2(2-\lambda)]}-\lambda(\epsilon+1-2(2-\lambda))}{2(1-\lambda)(\lambda+\epsilon-1)}
$$

- $\epsilon \geq 2 \frac{2-\lambda}{\lambda}-1: K_{\lambda \epsilon}<0$

- $\epsilon \in\left(1,2 \frac{2-\lambda}{\lambda}-1\right): K_{\lambda \epsilon} \notin \mathbb{R}$

- $\epsilon=1: K_{\lambda \epsilon}=1$

- $\epsilon \in(0,1): K_{\lambda \epsilon} \in\left(0 ; \bar{K}_{\epsilon}\right]$ with $\bar{K}_{\epsilon} \in\left(\frac{1}{4}, 1\right)$ that is reached in $\lambda_{\epsilon} \in\left(\frac{1}{3}, \frac{1}{2}\right)$, both of them increasing with $\epsilon$

It follows that linear contrats for which $K_{\lambda \epsilon} \in(0,1)$ are optimal for a Pareto distribution only if $\epsilon \in(0,1)$.

The necessity stems from the fact that plugging $z(\theta)=K \theta$ into Equation (9) gives appropriate restrictions on $f($.$) : indeed, one has A \theta \dot{f}(\theta)+B f(\theta)=0 \forall \theta$, which imposes that $f(\theta)=f_{0} \theta^{-\mu}$ with $\mu \equiv \epsilon+1$ and $f_{0}$ such that $f($.$) is a density over [\underline{\theta},+\infty)$, which yields the Pareto density.

The case when $B \equiv \epsilon \equiv 0$ corresponds to $f($.$) being constant, thus to uniformly dis-$ tributed valuations. In that case, $B=0 \Longleftrightarrow 2=(1-\lambda)(1-K)(2-\lambda+\lambda / K)$, which leads to $K_{\lambda}$ of Proposition 2. 


\section{References}

Bulow, J. (1982), 'Durable-goods monopolists', Journal of Political Economy 90(2), 314332 .

Coase, R. (1972), 'Durability and monopoly', Journal of Law and Economics 15(1), 143149 .

D'Haultfoeuille, X. \& Février, P. (2011), 'The provision of wage incentives: a structural estimation using contracts variation', Working paper CREST.

Güth, W. \& Ritzberger, K. (1998), 'On durable goods monopolies and the CoaseConjecture', Review of Economic Design 3, 215-236.

Gul, F., Sonnenschein, H. \& Wilson, R. (1986), 'Foundations of dynamic monopoly and the Coase conjecture', Journal of Economic Theory 39(1), 155-190.

Landsberger, M. \& Meilijson, I. (1985), 'Intertemporal price discrimination and sales strategy under incomplete information', RAND Journal of Economics 16(3), 424-430.

McAfee, R. P. \& Wiseman, T. (2007), 'Capacity Choice Counters the Coase Conjecture', Review of Economic Studies 75(1), 317-332.

Mirrlees, J. (1971), 'An exploration in the theory of optimum income taxation', Review of Economic Studies 38(2), 175-208.

Salant, S. (1989), 'When is inducing self-selection suboptimal for a monopolist?', The Quarterly Journal of Economics 104(2), 391-397.

Stokey, N. (1979), 'Intertemporal price discrimination', Quarterly Journal of Economics 93(3), 355-371.

Stokey, N. (1981), 'Rational expectations and durable goods pricing', Bell Journal of Economics 12(1), 112-128. 